For patients who were initially frequent exacerbators, moderate eosinophilia was associated with a sustained high exacerbation frequency $(p<0.05)$. High eosinophilia however was not associated with a sustained high exacerbation frequency $(\mathrm{p}=0.2178)$.

Of those patients with high eosinophilia $(n=65), 42.19 \%$ were on a high inhaled corticosteroid dose compared to $8.70 \%$ of those with moderate eosinophilia $(n=70),(p<0.0001)$.

Conclusion Our study shows that moderately raised eosinophils in COPD are associated with significantly increased long term exacerbation frequency trends.

Our data suggests that in clinical practice high eosinophil levels in COPD patients are more readily identified by clinicians, leading to escalation in ICS therapies to successfully manage exacerbation frequencies. The significance of moderate eosinophil levels is likely under recognised and these patients may also benefit from escalation in ICS therapies.

\section{S103 THE PROVISION OF THE FIVE FUNDAMENTALS OF COPD CARE - FINDINGS FROM A UK-WIDE SURVEY OF PEOPLE WITH COPD}

A Cumella, A Cook. The Asthma UK and British Lung Foundation Partnership, London, UK

\subsection{6/thorax-2021-BTSabstracts. 109}

Introduction and Aims Patient-reported data on care provided for people with chronic obstructive pulmonary disease (COPD) is patchy. This research aims to understand what care is being provided to this population, and how it affects people. This data will provide the basis of recommendations for service improvements.

Methods Between December 2020 - May 2021, the Asthma UK and British Lung Foundation ran an online survey of people with COPD. The survey received 8,232 responses. Using NICE's Five fundamentals of COPD care, ${ }^{1}$ respondents were asked about the care they received for their condition, as well as their experiences of living with the condition. Respondents were judged to have received the five fundamentals of care if they gave a positive answer to the elements they were eligible for (based on their MRC breathlessness score and smoking status).

Results $24.5 \%$ of all respondents received all of the measures of care they were entitled to. Considerable variation lies behind this overall figure, with rates of provision ranging from $28.5 \%$ in South West England, to $13.5 \%$ in Northern Ireland. $13.3 \%$ of respondents who told us they had more than 10 exacerbations in the past 12 months received these care measures, compared to $28.5 \%$ of people who between zero and two exacerbations in the past year. Those who have lived with COPD for over ten years were more likely $(36.0 \%$ received care measures) to receive these care measures than those who have been diagnosed in the past two years (10.6\%).

Conclusions This research indicates a significant proportion of people with COPD are not receiving the five fundamentals of COPD care. There is also considerable variation in provision among the COPD population, and improving care provision needs to be made a priority in order to improve clinical outcomes and reduce exacerbations.

\section{REFERENCE}

1. National Institute of Health and Care Excellence (NICE), 2018. Chronic obstructive pulmonary disease in over 16s: non-pharmacological management and use of inhaled therapies. Accessed at https://www.nice.org.uk/guidance/ng115/documents/supporting-documentation

\section{S104 COST-EFFECTIVENESS OF TRIPLE THERAPY WITH BUDESONIDE/GLYCOPYRRONIUM/FORMOTEROL FUMA- RATE VERSUS DUAL THERAPIES IN MODERATE-TO-VERY SEVERE COPD IN THE UNITED KINGDOM: ANALYSIS BASED ON THE KRONOS STUDY}

${ }^{1} \mathrm{E}$ de Nigris, ${ }^{2} \mathrm{U}$ Holmgren, ${ }^{3} \mathrm{C}$ Treharne, ${ }^{3} \mathrm{~N}$ Brighton, ${ }^{4} \mathrm{~A}$ Walker, ${ }^{5} \mathrm{~J}$ Haughney. ${ }^{1}$ Formerly of AstraZeneca, Cambridge, UK; ${ }^{2}$ AstraZeneca, Gothenburg, Sweden; ${ }^{3}$ Parexel International, London, UK; ${ }^{4}$ Salus Alba, Glasgow, UK; ${ }^{5}$ Clinical Research Facility, Queen Elizabeth University Hospital, Glasgow, UK

\subsection{6/thorax-2021-BTSabstracts.110}

Introduction The 24-week KRONOS study (NCT02497001) showed that fixed-dose triple therapy with budesonide/glycopyrronium/formoterol fumarate 320/14.4/10 $\mu \mathrm{g}$ (BGF 320) metered dose inhaler (MDI) was more efficacious at improving lung function than fixed-dose long-acting muscarinic antagonist (LAMA)/long-acting $\beta_{2}$-agonist (LABA) and inhaled corticosteroid (ICS)/LABA dual therapy in symptomatic patients with moderate-to-very severe chronic obstructive pulmonary disease (COPD). In KRONOS, $>70 \%$ of patients had no moderate or severe exacerbations in the previous year. Here, using KRONOS data, the lifetime cost-effectiveness of BGF 320 versus LAMA/LABA and ICS/LABA dual therapies in the United Kingdom is estimated.

Methods A Markov model was used to extrapolate incurred costs and quality-adjusted life-years (QALYs) for patients with moderate-to-very severe COPD. The model accounted for progression in severity (lung function decline), occurrence of moderate and severe exacerbations, adverse events, and discontinuations from the KRONOS study for BGF MDI 320, the LAMA/LABA glycopyrronium/formoterol fumarate 14.4/10 $\mu \mathrm{g}$ (GFF) MDI and the ICS/LABA budesonide/formoterol fumarate 400/12 $\mu \mathrm{g}$ (BUD/FORM) dry powder inhaler. Health care resource utilization was based on KRONOS data; unit costs came from UK National Health Service reference costs, the Personal Social Services Research Unit manual and the published literature. EuroQoL 5-dimension 5-level utilities for COPD severity states were estimated from KRONOS; exacerbation disutilities were sourced from a systematic literature review. A lifetime horizon was considered. Costs and QALYs were discounted at $3.5 \%$ per annum.

Results For BGF 320 versus GFF and BUD/FORM, respectively, incremental costs were $£ 1500$ and $£ 2598$, incremental QALYs were 0.49 and 0.38 and incremental exacerbation reductions were -4.29 and -1.78 . The incremental cost-utility ratios (ICUR) for BGF 320 were $£ 3082$ and $£ 6868$ per QALY gained versus GFF and BUD/FORM, respectively.

Discussion Based on KRONOS data, triple therapy with BGF 320 was cost-effective versus LAMA/LABA and ICS/LABA dual therapies at the conventional UK-adopted willingness to pay (ICUR < $£ 20000$ per QALY) in a moderate-to-very severe COPD population where $>70 \%$ of patients had no moderate or severe exacerbations in the prior year. The main cost-effectiveness driver for BGF 320 triple therapy versus dual therapies was the reduction in exacerbation rate, which reduced costs and preserved quality of life.

Please refer to page A190 for declarations of interest related to this abstract. 


\section{ILD: how big is the problem? How can you spot it and how should you monitor it?}

\section{P1 GLOBAL OVERVIEW OF INCIDENCE AND PREVALENCE OF INTERSTITIAL LUNG DISEASE: A SYSTEMATIC LITERATURE REVIEW}

R Gupta, A Koteci, A Morgan, PM George, JK Quint. Imperial College London, London, UK

\subsection{6/thorax-2021-BTSabstracts. 111}

Introduction Interstitial lung diseases (ILD) are a diverse group of pulmonary fibrotic and inflammatory conditions. The global burden of ILD is largely unknown, in part because of differences between countries in diagnosis and in coding practices ${ }^{1}$. Reviews to date have therefore tended to be limited in geographical scope and focused on individual ILDs. Our aim was to systematically review evidence for ILD prevalence and incidence on a global scale.

Methods A systematic search of Medline and Embase was conducted to identify relevant articles reporting the incidence and/or prevalence of individual ILD subtypes. The search was limited to observational studies published between 2015 and 2020 in English, with articles independently screened by two reviewers. An adapted Newcastle-Ottawa scale was used to assess quality and risk of bias.

Results Of 8,560 articles, 51 studies were included. Geographically, most studies were from Asia (47.1\%) and Europe

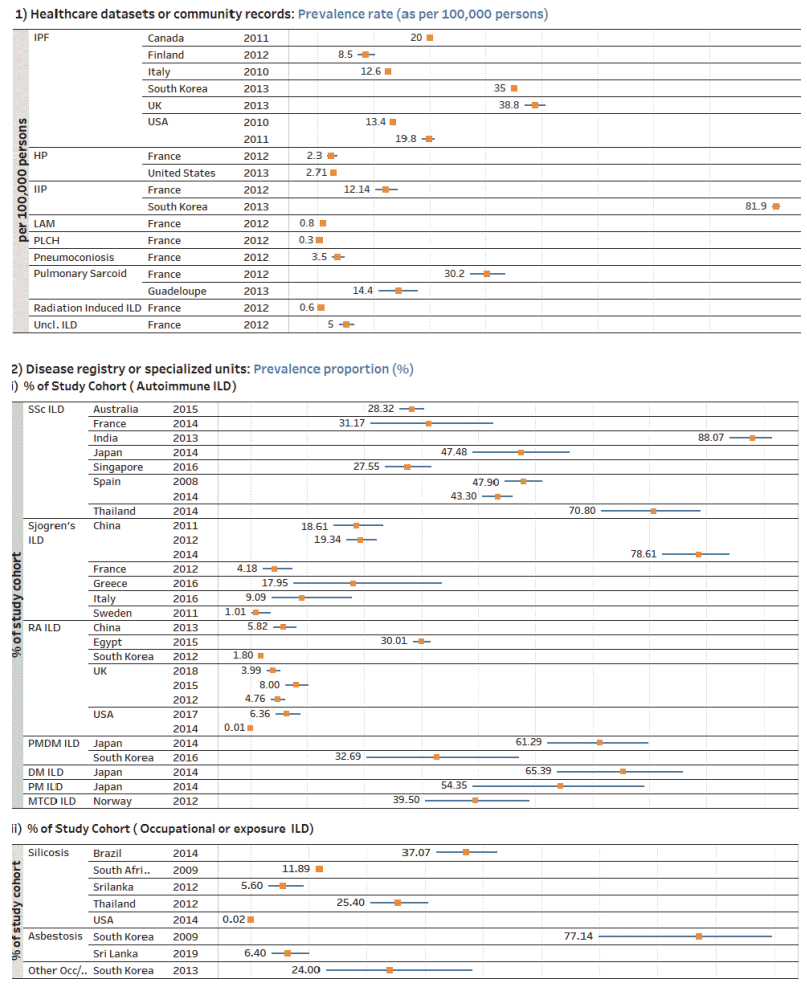

Abstract P1 Figure 1 Prevalence stratified by underlying population and reporting units

IIP, idiopathic interstitial pneumonia; IPF, idiopathic pulmonary fibrosis; NSIP, nonspecific interstitial idiopathic pneumonia; DIP, desquamative interstitial pneumonia; OP, organizing pneumonia; RA-ILD, rheumatoid arthritis ILD SSc-ILD, systemic sclerosis ILD; PM/ DM ILD, polymyositis/ dermatomyositis; MTCD, mixed connective tissue ILD; HP, hypersensitivity pneumonitis ILD; LAM, lymphangioleiomyomatosis; $\mathrm{PLCH}$, pulmonary Langerhans cell histiocytosis
(43.1\%). Significant heterogeneity was noted in the incidence and prevalence (figure 1) of ILDs between different countries. These variations are largely attributed to the diversity of the underlying population and differences in data sources used. For example, the prevalence of systemic sclerosis ILD ranged from $30 \%$ in Europe to $71 \%$ in Asia, silicosis ranged from $0.02 \%$ in the USA to $37 \%$ in Brazil. IPF was the most reported ILD, the range of prevalence was 8.5 to 38.8 per 100,000 persons, and incidence was 2.4 to 48.5 per 100,000 persons-years, across regions.

Discussion There have been few reviews investigating the epidemiology of ILDs overall or by subtypes. ILD publications differed by region, for example, more studies from Asia explored occupational ILDs, whereas more European studies reported on autoimmune ILDs. With an increasing research interest in progressive fibrosing ILDs, there is a need to understand the global burden of ILD and highlight unmet needs.

\section{REFERENCE}

1. King TE. Am J Respir Crit Care Med. 2005;172:268-279.

\section{P2 GEOGRAPHICAL VARIATION OF INTERSTITIAL LUNG DISEASE IN THE NORTHERN TRUST}

M Donaghy, S Gilmour, E Murtagh, P Minnis. Antrim Area Hospital, Interstitial Lung Disease Service, UK

\subsection{6/thorax-2021-BTSabstracts.112}

Aims There are geographical variations in Interstitial Lung disease, particularly sarcoidosis, that may shed light on aetiology and aid service development. The Northern Trust services a population of all most 471'000 people across a geographic area of 1,733 square miles making it the largest geographical trust in Northern Ireland. By exploring this unique attribute, we sought to define the geographical prevalence of Interstitial Lung Disease (ILD) and investigated whether this was affected by population density. Secondary aims included quality improvement in service provision.

Methods We identified 712 patients attending the ILD clinic within the last 12 months. Patients were classified on the basis of CT imaging and electronic care record review. Baseline demographic data and geographic location (Postcode) were collated and compared to recent census data to define population density and crude rates of prevalence. Average travel distance for patients attending clinic twice a year were calculated and compared to a proposed satellite clinic closer to patient homes.

Results Of the 712 patients included in analysis; $171(24 \%)$ Sarcoidosis, 157 (22.1\%) definite or probable UIP, 67 (9.4\%)

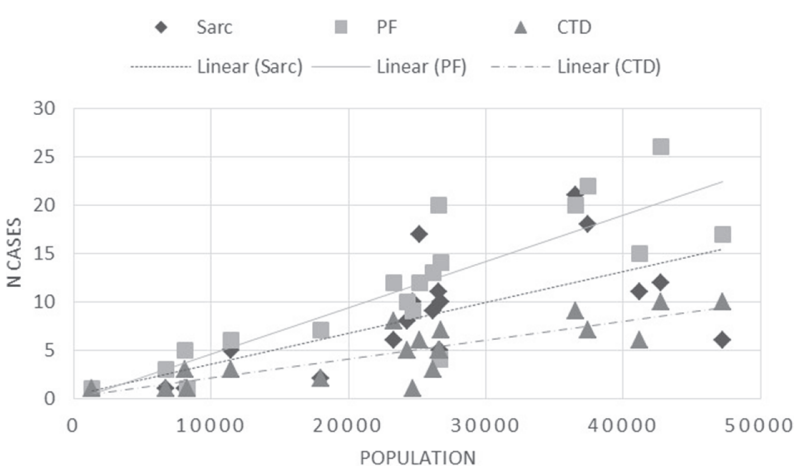

Abstract P2 Figure 1 